\title{
Model-Based Design and Optimization of Microfluidic Systems for Gentle Cellular Perfusion
}

\author{
Markus Gusenbauer ${ }^{1}$, Giulia Mazza ${ }^{1}$, Martin Brandl ${ }^{1}$, Thomas Schrefl, \\ Renáta Tóthová ${ }^{2}$ Iveta Jančigová ${ }^{2}$ Ivan Cimrák ${ }^{2}$ \\ ${ }^{1}$ Center for Integrated Sensor Systems, Danube University Krems, Austria \\ ${ }^{2}$ Department of Software Technologies, University of Zilina, Slovakia \\ markus.gusenbauer@donau-uni.ac.at
}

\begin{abstract}
Cell sensitive applications, like dynamic cell culturing, require the physiological perfusion of cell samples to the desired analysis slide. Stress occurring inside the flow chambers, connectors and pumping circuits can cause inflammatory reactions in blood cells or even cell-damage. Quantification of the mechanical stimuli with computer simulations enables the optimization of the system geometry, thus the minimization of the blood damage or activation. We use an automated procedure to calculate blood damage, regarding whole blood both as a continuum as well as a cell suspension. The obtained cell damage information feeds a mathematical optimization algorithm. The setup can be used to analyze and optimize arbitrary microfluidic systems for cell biological applications. Recent results of an optimized inlet of a flow chamber and a gentle pumping device are demonstrated.
\end{abstract}

Key words: CFD, blood cell modeling, blood damage index, cell activation, hemolysis

\section{Introduction}

Red Blood Cells (RBCs) are exposed to mechanical stimuli in the blood circulation. Changing the physiological environment can cause inflammatory reactions or even membrane rupture. These phenomena may start already by taking the blood sample (large change in pressure), followed by possible idle times (gravitational sinking) and induced shear forces by pumping systems, connectors and tubing. A controlled monitoring of the applied mechanical stress, especially in cell sensitive applications, like dynamic cell culturing, is mandatory. Non-physiological stimuli can change the blood cells expression and therefore bias experimental results.

Lysis of RBCs (breakage of cell membrane) depends on the exposure time of an applied shear stress [1]. Large shear stress $(1,000$ $\left.\mathrm{N} / \mathrm{m}^{2}\right)$ can only be applied for a short time $(0.1$ $\mathrm{ms}$ ) before damaging the cell membrane. On the other side, long exposure times (100 s) with lower shear stress $\left(150 \mathrm{~N} / \mathrm{m}^{2}\right)$ may also damage the cells. According to [2] the traumata of blood cells in pumping systems can be quantified using parameters like free Hemoglobin (fHb), platelets count (PLT), erythrocyte fragility (EF) and the morphological analysis of erythrocytes under the microscope.
fHB and PLT increase linearly with the pumping time (long term exposure).

In the development of blood pumps and similar artificial circuits, the damage on blood has been investigated thoroughly (e.g. [3]). We developed a sensing platform for cell damage and/or cell activation in microfluidic systems [4]; these features are difficult to characterize with the standard analysis methods, due to very small blood volumes and long perfusion times.

\section{Numerical Optimization}

In the design process of medical devices, finite element analysis and numerical optimization are more and more useful instruments $[5,6]$. With the use of numerical optimization tools, an optimal design is determined based on a structured and automated process. Opposed to a manual design process, in which the engineer makes decisions based on intuition and experience, a mathematical optimization algorithm determines the design parameters. A widely used objective function is the maximum principal strain. A recent example is the computational design of a vascular stent [6].

Our optimization routine applies a blood damage index (BDI) and cell damage index (CDI) as objective functions (Fig. 1). The BDI is used to approximate the occurring hemolysis in a fluidic regime. Navier-Stokes fluid dynamics 
are computed to obtain the velocity field (ElmerFEM, www.csc.fi/web/elmer, last visited on 29/03/2017), from which a power law based equation estimates the cell lysis (Eqn. 1).

$$
B D I=\frac{1}{N_{\text {line }}} \sum_{\text {lines }} \sum_{\text {inlet }}^{\text {outlet }} C \Delta t^{a} \tau^{b}
$$

The power law constants $C, a$ and $b$ have to be fitted to the fluid and device characteristics, as commonly done in the investigation of blood pumping circuits (e.g. [7]). Streamlines are built from the computed velocity field (ParaView, www.paraview.org, last visited on 29/03/2017) to sum the occurring shear stress $\tau$ over small time steps $\Delta t$ (Fig. 2a).

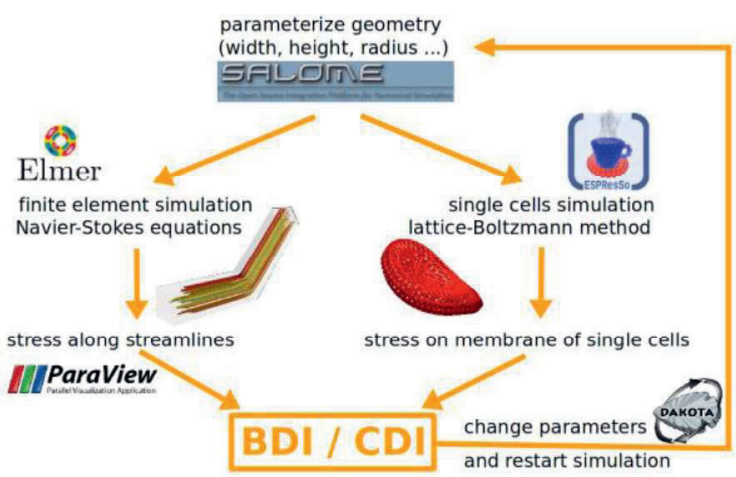

Fig. 1. Automated optimization routine to minimize the blood damage in microfluidic systems.

As alternative to the continuum fluid dynamics simulations for whole blood, we compute individual cells as well. The blood cells, immersed in a lattice-Boltzmann fluid dynamics code, are modeled using an elastic surface membrane, and validated with stretching experiments [8] (Fig. 2b,c).
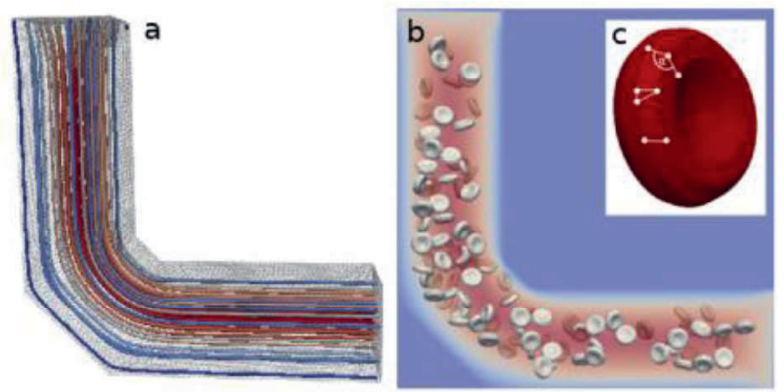

Fig. 2. Comparison of simulation methods in bent microfluidic channel: Streamlines are computed with a Navier-Stokes velocity field (a) and single cells are immersed in a lattice-Boltzmann grid (b). The blood cell consists of calibrated constraints on the membrane to allow elasticity and keep the original shape (c).

The damage on one cell is calculated as the sum of relative cell surface area changes during the passage through the device (current $S$ and original surface area $S_{0}$, Eqn. 2).

$$
C D I=\frac{1}{N_{\text {cells }}} \sum_{\text {cells }} \sum_{\text {inlet }}^{\text {outlet }}\left(S-S_{0}\right) / S_{0}
$$

The damage of each individual cell is averaged to get a single CDI value for the objective function.

Both, the BDI and CDI are feeding the numerical optimization tool (Dakota, dakota.sandia.gov, last visited on 29/03/2017), which changes the parameterized geometry in our geometry generation (meshing) tool (Salome, www.salome-platform.org, last visited on 29/03/2017). All software tools used in the automated optimization routine are open source.

\section{Intracellular calcium}

In addition to the computational workflow, we established an experiment setup to monitor mechanical stimulation on cells using fluorescent calcium indicators. As already reported for leukocytes [9], RBCs react with a rise in intracellular calcium when subjected to increased shear stress [10]. Bright field and fluorescence field videos are recorded to see the time response of the calcium in microfluidic pathways (Fig. 3). Pixel intensities of the fluorescence video can be automatically evaluated to detect frequency and amplitude of the calcium transients.
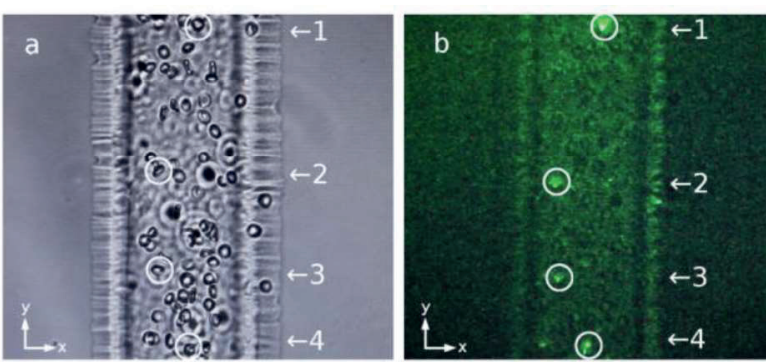

Fig. 3. Bright field (a) and fluorescence field (b) snapshots of a microfluidic cell flow. Calcium transients are highlighted.

\section{Optimizing inlet of flow chambers}

A common problem found in flow chambers for live cell imaging (Fig. 4a), is the possible turbulence created at the inlets. The cells are exposed to large mechanical stress, due to the sudden cross section change and the connections perpendicular to the analysis slide. Using our proposed optimization routine, we were able to reduce the mechanical stress at the inlets by approximately $43 \%$ (Fig. 4b). We still demanded a perpendicular connection of tubing, but allowed some free parameters of angles and radii. 

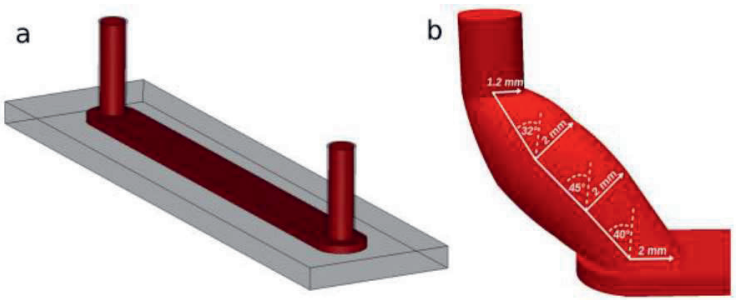

Fig. 4. Optimization of flow chamber inlets (a): $A$ reduction of about $43 \%$ of mechanical stress on cells can be achieved with the new design (b).

\section{Cell sensitive pumping}

Based on the pumping principle of Hatch et al. [11] we built and optimized a pumping system with reduced cell activation (Fig. 5) [12]. Two spherical NdFeB plugs are driven by electromagnets. Plug $A$ is temporarily fixed to block the short path between inlet and outlet, while Plug $B$ is pushing the fluid. After one cycle plug $B$ swaps the position with plug $A$ and the cycle starts again.
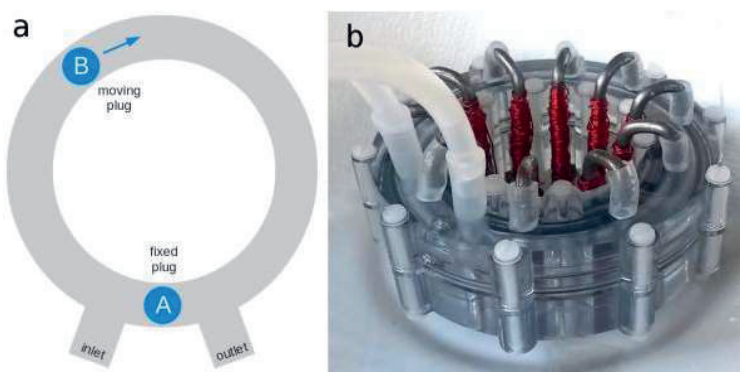

Fig. 5. Principle (a) and final prototype (b) of our developed gentle cellular pumping device. Two $\mathrm{NdFeB}$ plugs ( $A$ and $B$ ) are anti-cyclically driven by electromagnets.

A key parameter in the optimization of the pumping device is the size of the gap between the moving plugs and the channel wall. Allowing

\section{References}

[1] S. Deutsch et al., Experimental fluid mechanics of pulsatile artificial blood pumps. Annu. Rev. Fluid Mech., 38, 65-86 (2006); doi: 10.1146/ annurev.fluid.38.050304.092022

[2] M. J., Ding et al, Trauma to erythrocytes induced by long term in vitro pumping using a roller pump. Cell biology international, 31(8), 763-767 (2007); doi: 10.1016/j.cellbi.2007.01.030

[3] S. R Shah et al, CFD for centrifugal pumps: a review of the state-of-the-art. Procedia Engineering, 51, 715-720 (2013); doi: 10.1016/j.proeng.2013.01.102

[4] M. Gusenbauer et al., Sensing Platform for Computational and Experimental Analysis of Blood Cell Mechanical Stress and Activation in Microfluidics. Procedia Engineering, 168, 13901393 (2016); doi: 10.1016/j.proeng.2016.11.388 a certain leakage and fluid flow reduces the stress on blood cells, but also reduces the performance of the pump. The final device (Fig. $5 b)$ was printed using the Stratasys $J 7503 \mathrm{D}$ printer (Stratasys Ltd, USA) for fast-prototyping.

\section{Summary}

In this work, we have demonstrated a setup for sensing the mechanical stress on blood cells. A computational model of single RBC delivers information on the stretching of the cell membrane. In addition simulations of continuum Navier-Stokes fluid dynamics provide information on the shear stress in whole blood. Both methods were supported by experimental quantification of rising intracellular calcium levels, which are shear stress dependent.

We used an automated optimization routine to minimize cell activation (and damage) in flow chambers for live cell imaging. Furthermore, we developed a pumping system for reduced cell activation. Both examples illustrate our main goal to physiologically treat blood cells outside the human circulatory system. In other words, we would like to keep the blood cells happy and alive.

\section{Acknowledgement}

The authors gratefully acknowledge the financial support of the NÖ Forschungs- und Bildungsges.m.b.H. (NFB) through the Life Science Calls (Project ID: LSC13-024). Ivan Cimrák, Iveta Jančigová and Renáta Tóthová were supported by the Slovak Research and Development Agency (contract number APVV15-0751). We thank the research group of Bernd Bickel at Institute of Science and Technology Austria for 3D printing of the device.

[5] M.I. Kilani, M.I. Al-Widyan, A. Al Halhouli, Analytical and computational fluid dynamics (CFD) investigation of the applicability of a ferrofluidic magnetic micropump for fluids with stress-sensitive mircroparticles, Scientific Research and Essays 8.19: 754-766 (2013); doi: 10.5897/SRE2013.5370

[6] Red Cedar Technology, White Paper 1024, Optimial Design of Medical Devices, www.redcedartech.com/pdfs/Optimal_Design_of_ Medical_Devices.pdf, last visited on 29/03/2017

[7] M. E. Taskin et al., Evaluation of eulerian and lagrangian models for hemolysis estimation, ASAIO journal, 58, 363-372 (2012); doi: 10.1097/MAT.0b013e318254833b

[8] I. Cimrák, M. Gusenbauer, T. Schrefl, Modelling and simulation of processes in microfluidic devices for biomedical applications. Computers \& 
Mathematics with Applications 64.3, 278-288

(2012); doi: 10.1016/j.camwa.2012.01.062

[9] G. J. Zwartz et al., A miniature couette to generate shear for flow cytometry: studying realtime modulation of intracellular calcium in monocytic cells, Cytometry A, 79, 233-40 (2011); doi: 10.1002/cyto.a.21027

[10] A. Bogdanova et al., Calcium in red blood cells a perilous balance, International journal of molecular sciences, 14, 9848-9872 (2013); doi: 10.3390/ijms 14059848

[11] A. Hatch et al., A ferrofluidic magnetic micropump, Journal of Microelectromechanical systems, 10 (2), 215-221 (2001); doi: $10.1109 / 84.925748$

[12] M. Gusenbauer et al., Magnetically actuated circular pump, Submitted to International Journal of Heat and Fluid Flow. (2017) 